\title{
REAL-ANALYTIC SUBMANIFOLDS OF COMPLEX MANIFOLDS
}

\author{
L. R. HUNT
}

Abstract. This paper examines the extendibility of holomorphic functions on a real manifold which is embedded in a complex manifold. The principal result is that all real $k$-dimensional, real-analytic, compact manifolds embedded in an $n$-dimersional complex Stein manifold, where $k>n$, are extendible over a manifold of one higher real dimension. A discussion is also given of the local equations of a manifold which is C-R in a neighborhood of some point.

1. Introduction. Wells [3] proves that there is an open dense set of embeddings of a real $k$-dimensional $\mathfrak{C}^{\infty}$ compact manifold into an $n$-dimensional complex manifold $X, k>n$, with the property that each submanifold of $X$ embedded by an element in this dense set is extendible over a real $(k+1)$-dimensional $\mathfrak{e}^{m}$ manifold, $1 \leqq m<\infty$. It is the purpose of this paper to show that all real $k$-dimensional, real-analytic, compact manifolds embedded in an $n$-dimensional complex Stein manifold $X, k>n$, are extendible over a manifold of one higher real dimension.

In $\$ 2$ of this paper we give definitions of exceptional points, extendibility, and the Levi form. $\S 3$ contains a discussion of the local equations of a manifold which is $\mathrm{C}-\mathrm{R}$ in a neighborhood of some point. A short discussion of peak points is given in $\$ 4$, and we prove our result for real-analytic submanifolds of complex manifolds in $\$ 5$.

2. Definitions. Let $M^{k}$ be a real $k$-dimensional $\mathfrak{e}^{\infty}$ manifold embedded in an $n$-dimensional complex manifold $X$, where $k, n \geqq 2$. If $f$ is the embedding, then the real Jacobian of $f$ has maximal rank. Let us denote the complex Jacobian of $f$ by $J(f)$ and set $q=\min (n, k)$. A point $p$ in $M^{k}$ is said to be an exceptional point of order $l, 0 \leqq l$ $\leqq[k / 2]-\max (k-n, 0)$, if the complex rank of $\left.J(f)\right|_{p}$ is equal to $q-l$.

A point $p$ in $M^{k}$ is generic if $p$ is an exceptional point of order 0 . The manifold $M^{k}$ is locally generic at $p$ in $M^{k}$ if every point in some open neighborhood of $p$ is generic, and is locally $C-R$ at $p$ if every point in some open neighborhood of $p$ is an exceptional point of the

Received by the editors August 13, 1970.

AMS 1969 subject classifications. Primary 3220, 3225; Secondary 3227.

Key words and phrases. Extendibility of holomorphic functions, real-analytic submanifolds of complex manifolds, exceptional points, locally C-R. 
same order. If $k \leqq n$, an exceptional point of order 0 is a totally real point.

Suppose $M^{k}$ is locally C-R at $p$, and $p$ is not a totally real point for $M^{k}$. Let $T_{x}\left(M^{k}\right)$ be the real tangent space to $M^{k}$ at $x$, and $H_{x}\left(M^{k}\right)$ be the space of holomorphic tangent vectors to $M^{k}$ at $x$. Then we define the Levi form, at any $x$ near $p$,

$$
L_{x}\left(M^{k}\right): H_{x}\left(M^{k}\right) \rightarrow\left(T_{x}\left(M^{k}\right) \otimes C\right) /\left(H_{x}\left(M^{k}\right) \otimes C\right)
$$

by $L_{x}\left(M^{k}\right)(t)=\pi_{x}\left\{[Y, \bar{Y}]_{x}\right\}$, where $Y$ is a local section of $H\left(M^{k}\right)$ such that $Y_{x}=t,[Y, \bar{Y}]_{x}$ is the Lie bracket evaluated at $x$, and

$$
\pi_{x}: T_{x}\left(M^{k}\right) \otimes C \rightarrow\left(T_{x}\left(M^{k}\right) \otimes C\right) /\left(H_{x}\left(M^{k}\right) \otimes C\right)
$$

is the projection.

Denote by $\mathcal{O}_{x}=\mathcal{O}$ the sheaf of germs of holomorphic functions on the second countable complex manifold $X$. Let $K$ be a compact subset of $X$ and $U$ an open subset of $X$ containing $K$. We set $\mathcal{O}(K)=$ ind $\lim _{U \supset K} \mathcal{O}(U)$ where $\mathcal{O}(U)$ is the Fréchet algebra of holomorphic functions on $U$. We say that $K$ is extendible to a connected set $K^{\prime}{ }_{\neq} K$ if the map $r: \mathcal{O}\left(K^{\prime}\right) \rightarrow \mathcal{O}(K)$ is onto.

3. Local equations. Let $J^{r}\left(M^{k}, X\right)$ be the $r$-jet bundle with fiber $J^{r}(k, n)$ (e.g. see [2]). We can identify $J^{1}(k, n)$ with the set of all complex $(n \times k)$ matrices. Denote by $S_{l}(k, n)=S_{l}$ the subset of $J^{1}(k, n)$ with rank equal to $(q-l), 0 \leqq l \leqq[k / 2]-\max (k-n, 0)$. Then if $M^{k}$ is embedded in $X$ by the map $f$, a point $p$ in $M^{k}$ is an exceptional point of order $l$ if and only if $\left.J(f)\right|_{p}$ is in $S_{l}$.

Suppose the point $p$ in $M^{k}$ is an exceptional point of order $l$. If $k \geqq n$, then the local equations of $M^{k}$ in a neighborhood of $p$ are given (after a proper coordinate change) by

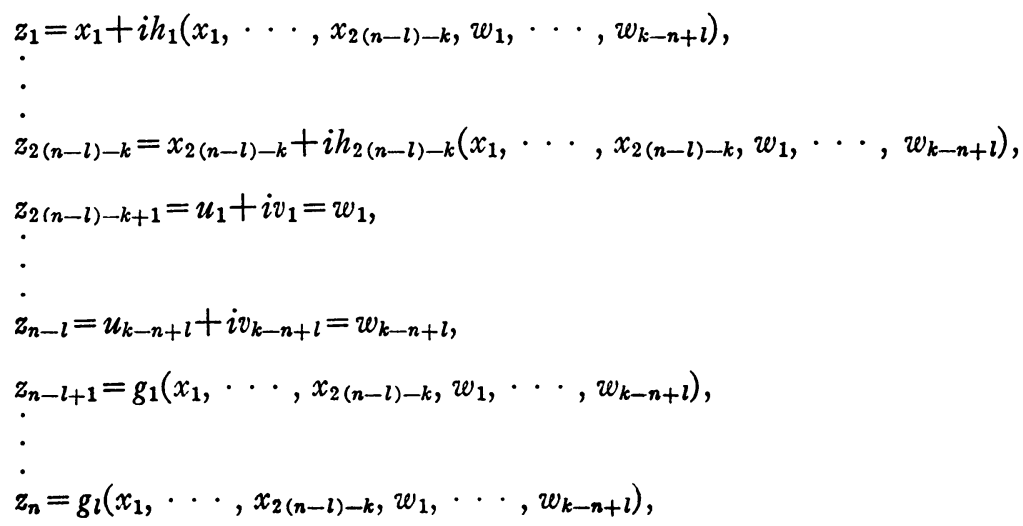


where $x_{1}, \cdots, x_{2(n-l)-k}, u_{1}, v_{1}, \cdots, u_{k-n+l}, v_{k-n+l}$ are local coordinates for $M^{k}$ in a neighborhood of $p$ vanishing at $p$, and $z_{1}, \ldots$, $z_{n}$ are local coordinates for $X$ in a neighborhood of $p$ vanishing at $p$. The real-valued functions $h_{1}, \cdots, h_{2(n-l)-k}$ as well as the complexvalued functions $g_{1}, \cdots, g_{l}$ vanish to order 2 at $p$.

In [2] we state that $S_{l}$ is a regular submanifold of $J^{1}(k, n)$ of complex codimension $(k-n+l) l$. However, we notice that $(k-n+l) l$ is just the number of complex lines in the local equations times the number of functions $g_{i}$ in the local equations.

The question we are interested in is the following. What form must the equations take in order for the embedded manifold to be locally C-R at the exceptional point $p$ of order $l$ ? Since every point $p^{\prime}$ in some open neighborhood of $p$ would have the property that $\left.J(f)\right|_{p^{\prime}}$ must satisfy $(k-n+l) l$ complex conditions, we find it is difficult (in the sense of transversality theory) for a manifold to be locally C-R except when $l=0$. Using equations (1) we find that our complex Jacobian at a point $p^{\prime}$ can be put in the form

$$
\left[\begin{array}{ll}
A & B \\
C & D
\end{array}\right]
$$

where $A$ is the $(2(n-l)-k) \times(2(n-l)-k)$ identity matrix, $B$ and $C$ are both zero matrices, and $D$ is the $(2 l+k-n) \times(2(k-n+l))$ matrix

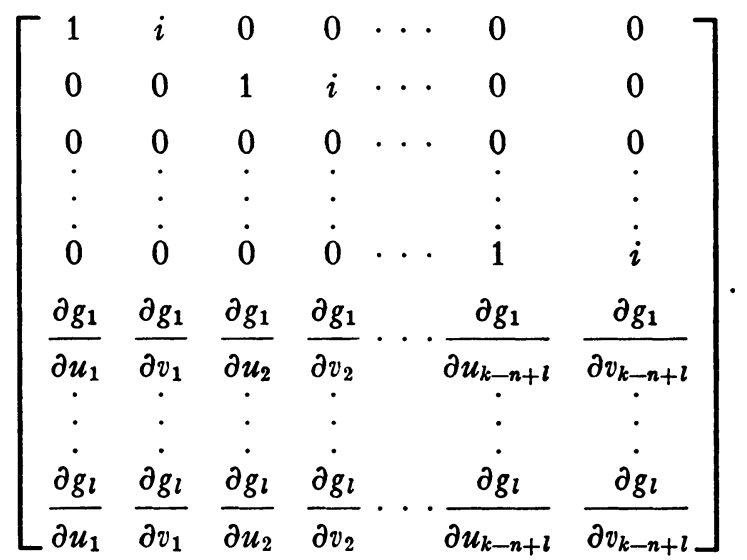

Hence $M^{k}$ is locally C-R at the exceptional point $p$ of order $l$ if and only if $\left(\partial g_{i} / \partial u_{j}\right)=-i\left(\partial g_{i} / \partial v_{j}\right)$ holds in some neighborhood of $p$ for $j=1, \cdots, k-n+l$ and $i=1, \cdots, l$. Thus for the manifold $M^{k}$ to be locally C-R the functions $g_{1}, \cdots, g_{l}$ in our local equations (1) must be complex-analytic functions of $w_{1}, \cdots, w_{k-n+l}$. 
Again let $p$ be an exceptional point of order $l$ in $M^{k}$, but assume $k<n$. The local equations of $M^{k}$ in a neighborhood of $p$ are given (after a proper coordinate change) by

$$
\begin{aligned}
& z_{1}=x_{1}+i h_{1}\left(x_{1}, \cdots, x_{k-2 l}, w_{1}, \cdots, w_{l}\right), \\
& \vdots \\
& z_{k-2 l}=x_{k-2 l}+i h_{k-2 l}\left(x_{1}, \cdots, x_{k-2 l}, w_{1}, \cdots, w_{l}\right), \\
& z_{k-2 l+1}=u_{1}+i v_{1}=w_{1}, \\
& \vdots \\
& z_{k-l}=u_{l}+i v_{l}=w_{l}, \\
& z_{k-l+1}=g_{1}\left(x_{1}, \cdots, x_{k-2 l}, w_{1}, \cdots, w_{l}\right), \\
& \dot{z_{n}}=g_{n-k+l}\left(x_{1}, \cdots, x_{k-2 l}, w_{1}, \cdots, w_{l}\right),
\end{aligned}
$$

where $x_{1}, \cdots, x_{k-2 l}, u_{1}, v_{1}, \cdots, u_{l}, v_{l}$ are the local coordinates for $M^{k}$ in a neighborhood of $p$ vanishing at $p$, and $z_{1}, \cdots, z_{n}$ are the local coordinates for $X$ in a neighborhood of $p$ vanishing at $p$.

We have that $S_{l}$ is a regular submanifold of $J^{1}(k, n)$ of complex codimension $(n-k+l) l$. In order for the manifold to be locally $\mathrm{C}-\mathrm{R}$ at $p$, a discussion similar to the previous one tells us that the functions $g_{1}, \cdots, g_{n-k+l}$ in our local equations (2) must be complexanalytic functions of $w_{1}, \cdots, w_{l}$.

4. Peak points and the Levi form. Let $K$ be a compact subset of a complex manifold $X$. We call a point $x \in K$ a holomorphic peak point if there exists a function $f \in \mathcal{O}(K)$ such that, for any $y \in K-\{x\}$, we have $|f(y)|<|f(x)|$.

In [3] it is shown that a compact $\mathfrak{C}^{2}$ submanifold of a Stein manifold $X$ contains a nonempty open set of peak points. Also the Levi form cannot vanish at a peak point without contradicting the maximum principle.

5. Real-analytic manifolds. In this section we shall prove the asserted result for real-analytic manifolds.

THEOREM 5.1. Let $M^{k}$ be a real $k$-dimensional, real-analytic, compact manifold embedded in an $n$-dimensional Stein manifold $X, k>n$. Then $M^{k}$ is extendible to a $\mathrm{e}^{m}$ submanifold $\tilde{M}$ of $X$ with real dimension $\tilde{M}=k+1$ and $1 \leqq m<\infty$.

Proof. Given any point $p$ in $M^{k}$, arbitrarily close to $p$ we can find an open set of points at which $M^{k}$ is locally C-R. This follows from 
the fact that if $M^{k}$ is not locally C-R at $p$, then arbitrarily close to $p$ we can find a point which is an exceptional point of order less than the order of $p$. We repeat this process until we find a point at which $M^{k}$ is locally C-R (for a "dense" set of embeddings this will mean locally generic).

Let $p$ be a peak point in the open set mentioned in $\$ 4$. Then there exists a point $p^{\prime}$ in $M^{k}$ and an open neighborhood of this point on which $M^{k}$ is locally C-R and the Levi form does not vanish. If $M^{k}$ is locally generic at $p^{\prime}$, we have the conditions for local extendibility given in [1] and [4] and the proof is complete. If $M^{k}$ is locally C-R at $p^{\prime}$ and not generic there we have the local equations (1) where $l>0$ and $g_{1}, \cdots, g_{l}$ are complex-analy tic functions of $w_{1}, \cdots, w_{k-n+l}$.

Since our manifold is real-analytic, we expand each function $g_{i}$ in a power series

$$
g_{i}(x, w)=\sum_{\alpha} a_{i, \alpha}(x, w)^{\alpha}
$$

where $\quad x=\left(x_{1}, \cdots, \quad x_{2(n-l)-k}\right), \quad w=\left(w_{1}, \cdots, \quad w_{k-n+l}\right), \quad$ and $\alpha=\left(\alpha_{1}, \cdots, \alpha_{n-l}\right)$. Replacing $z_{j}$ by $z_{j}-\sum_{\alpha} a_{i, \alpha} z^{\alpha}$, where $z=\left(z_{1}, \cdots, z_{n-l}\right), j=n-l+1, \cdots, n$, and $j=n-l+i$, we find in our new coordinates that each $a_{i, \alpha}=0$. Hence our local equations in our new coordinate system are

(3)

$$
\begin{aligned}
& z_{1}=x_{1}+i h_{1}\left(x_{1}, \cdots, x_{2(n-l)-k}, w_{1}, \cdots, w_{k-n+l}\right), \\
& \dot{\cdot} \\
& z_{2(n-l)-k}=x_{2(n-l)-k}+i h_{2(n-l)-k}\left(x_{1}, \cdots, x_{2(n-l)-k}, w_{1}, \cdots, w_{k-n+l}\right), \\
& z_{2(n-l)-k+1}=u_{1}+i v_{1}=w_{1}, \\
& : \\
& \dot{z}_{n-l}=u_{k-n+l}+i v_{k-n+l}=w_{k-n+l}, \\
& z_{n-l+1}=0 \\
& \dot{:} \\
& \dot{z}_{n}=0 . \\
&
\end{aligned}
$$

Thus our point $p^{\prime}$ appears locally as a generic point of a real $k$ dimensional submanifold of $C^{n-l}$. Since the Levi form's vanishing or nonvanishing is independent of coordinate changes, we have a generic point at which the Levi form does not vanish. This establishes the extendibility as given in the statement of the theorem.

REMARK. The theorem is false if $k \leqq n$ because of the existence of totally real submanifolds which are always holomorphically convex. 


\section{REFERENCES}

1. S. J. Greenfield, Cauchy-Riemann equations in several variables, Ann. Scuola Norm. Sup. Pisa (3) 22 (1968), 275-314. MR 38 \#6097.

2. L. R. Hunt, Exceptional points of a differentiable submanifold of a complex manifold (to appear).

3. R. O. Wells, Jr., Concerning the envelope of holomorphy of a compact differentiable submanifold of a complex manifold, Ann. Scuola Norm. Sup. Pisa (3) 23 (1969), 347361. MR $39 \# 7141$.

4. - Holomorphic hulls and holomorphic convexity of differentiable submanifolds, Trans. Amer. Math. Soc. 132 (1968), 245-262. MR 36 \#5392.

Texas Tech University, Lubbock, Texas 79409 